Article

\title{
Differential Service in a Bidirectional Radio-over-Fiber System over a Spectral-Amplitude-Coding OCDMA Network
}

\author{
Chao-Chin Yang ${ }^{1}$, Kai-Sheng Chen ${ }^{2, *}$, Jen-Fa Huang ${ }^{2}$ and Jia-Cyuan Kuo ${ }^{2}$ \\ 1 Department of Electro-Optical Engineering, Kun Shan University, Tainan 701, Taiwan; ccyang@ksu.edu.tw \\ 2 Insitute of Computer and Communication Engineering, Department of Electrical Engineering, National \\ Chen Kung University, Tainan 710, Taiwan; huajf@ee.ncku.edu.tw (J.-F.H.); cyuan0912@gmail.com (J.-C.K.) \\ * Correspondence: weiweibjqskwx@hotmail.com; Tel.: +886-926-887-654
}

Received: 31 August 2016; Accepted: 5 October 2016; Published: 18 October 2016

\begin{abstract}
A new scheme of radio-over-fiber (RoF) network based on spectral-amplitude-coding (SAC) optical code division multiple access (OCDMA) is herein proposed. Differential service is provided by a power control scheme that classifies users into several classes and assigns each of them with a specific power level. Additionally, the wavelength reuse technique is adapted to support bidirectional transmission and reduce base station (BS) cost. Both simulation and numerical results show that significantly differential quality-of-service (QoS) in bit-error rate (BER) is achieved in both downlink and uplink transmissions.
\end{abstract}

Keywords: radio-over-fiber (RoF); spectral amplitude coding (SAC); differential service; wavelength reuse

\section{Introduction}

Radio-over-fiber (RoF) supports broadband interconnections between base stations (BS's) and the central office (CO) to release the heavy burden of wireless throughput [1-4]. For an adequate RoF transmission, multiplexing techniques are applied to both optical and electrical domains. Optical time-division multiple access (TDMA) and wavelength-division multiple access (WDMA) are main schemes to multiplex signals from different users. For TDMA, users are allocated in different time slots. When the user number increases, the queue time between two successive transmissions becomes longer. Additionally, there is a stringent requirement of synchronization between transmitter and receiver. WDMA has a simple structure and fairly increases the transmission rate, but it is not efficient in bandwidth utilization, since only one wavelength can be used by a certain user. On the other hand, optical code-division multiple-access (OCDMA), providing multiple users with asynchronous access without scheduling, is a promising solution to implementing RoF [2-4]. Spectral amplitude coding (SAC) has been studied in many RoF schemes $[3,4]$ since it is free from multiple access interference (MAI), and the data can be encoded on an optical carrier without pre-sampling.

In this paper, we propose a new system including two novel characteristics: supporting multi-service transmission and adapting wavelength reuse structure. These two issues have not been studied in previous research on SAC-based RoF. Due to the variant data traffics and diverse service types, scheduling algorithms on a wireless [5] and processing control scheme on an optical signal [6] is proposed to provide the requested quality of service (QoS). The optical control scheme is preferable because of its short processing time and low cost.

We propose a power control scheme that classifies the total users into several classes. Different power levels are assigned to each class according to the specific class-of-service. Figure 1 shows the user classification of a 2-class scheme. $K_{i}$ and $P_{i}$ is the number of users and power in class $i$, respectively, 
where $i=1,2$. The power level $P_{2}$ is higher than $P_{1}$, so a higher signal-to-noise ratio (SNR) is detected for Class 2 and better service is achieved. Furthermore, the desired user can be identified through MAI elimination even if all coded signals are multiplexed in the same wavelength band.

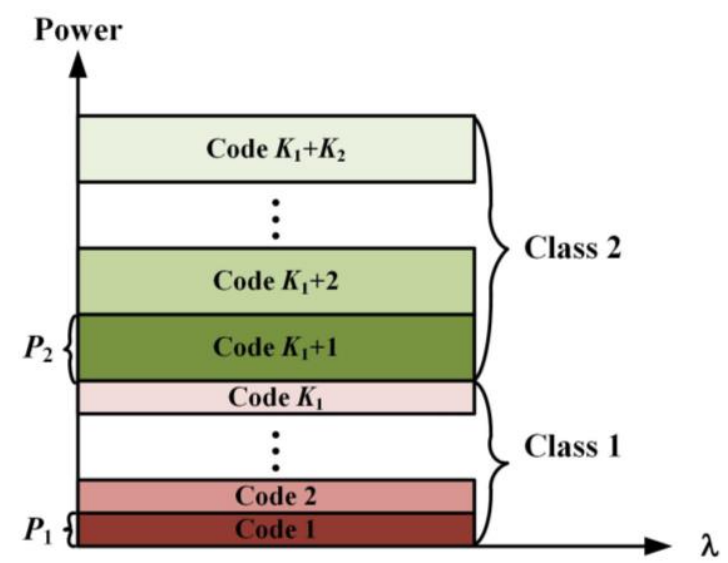

Figure 1. User classification and power distribution in the proposed differential service system with spectral amplitude coding (SAC) optical code-division multiple-access (OCDMA).

Our second contribution is the designation of a cost-effective BS. Wavelength reuse is commonly used for reducing the cost of a RoF network [7,8], where both downlink and uplink share the same light source. This has advantages such as the centralization of the light source at the $\mathrm{CO}$ and a simplified BS structure. However, the broadband light source (BLS) used for SAC encoding is not suitable for generating the optical millimeter, which is required by most wavelength reuse schemes. A coherent light source, generally a laser, is used to generate optical millimeters for up- and downlink transmissions by modulation schemes such as a double side band (DSB) [9] or a single side band (SSB) [10]. Unlike a laser with a near impulse spectrum, the broadband light source (BLS) for SAC encoding occupies a range of wavelength band of 1 to $10 \mathrm{THz}$. Employing DSB or SSB on BLS would result in an overlapping between the down- and uplink carriers. Thus, in this paper, we employ the concept of wavelength-division multiplexing (WDM). The spectrum of BLS from the CO is divided by a de-multiplexer (De-Mux) into two carriers. Only one of the carriers is modulated by the downlink data, while the other is for the uplink transmission.

To investigate the proposed system performance, the bit-error rate (BER) is used to quantify QoS by considering the effects of thermal noise and phase-induced intensity noise (PIIN). Along with simulations, the numerical results show that we achieve significantly differential service between individual users when employing power control in the proposed RoF architecture.

\section{The Proposed Bi-Directional RoF System with Power Control Scheme}

The architecture of the bi-directional SAC-based RoF along with the power control and wavelength reuse is shown in Figure 2. For simplicity, there are only two classes, and each of them has one user in it. As for Class 1, BLS\#1 is separated into two optical carriers by a De-Mux. Downlink passband signal is modulated on the first carrier by intensity modulation (IM). Then, the modulated carrier is sent to the SAC encoder employed in [11] for encoding. An optical coupler (OC) combines the coded carrier with the un-modulated one to obtain the downlink signal. The downlink signal of Class 2 is similar to that of Class 1, except for the code vector assigned to each user and the power level of the BLS. Before being transmitted to the BS, downlink signals from Class 1 and Class 2 are combined again by another OC. 


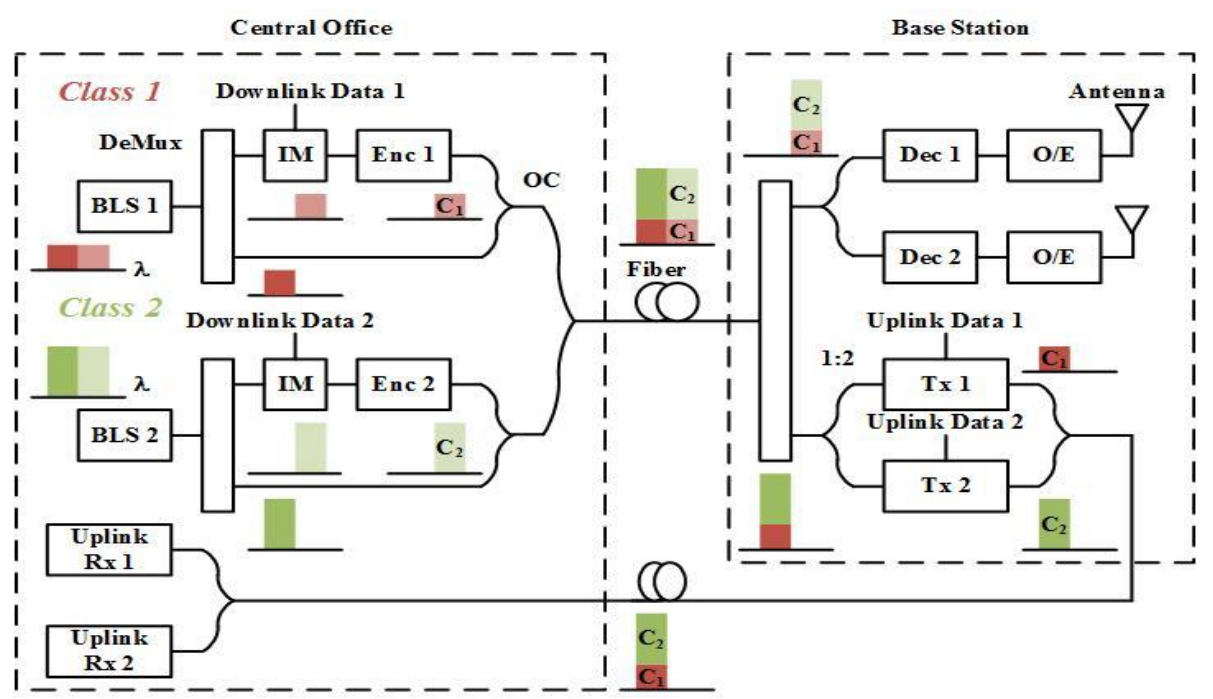

Figure 2. Configuration of the wavelength-reused radio-over-fiber (RoF) network with power control technique.

We use a modified stuffed-shift prime code (MSSP code) [12] in the proposed OCDMA-based RoF system. A MSSP code has the following properties: $M=p^{2}+p+1, \omega=p+1$, and $\lambda=1$, where $M$ is the code length, $\omega$ is the code weight, $\lambda$ is the cross-correlation, and $p$ is a prime number. Two MSSP codes of $p=2-(1,0,1,0,1,0,0)$ and $(0,1,0,1,1,0,0)$-are assigned to user\#1 and user\#2, respectively. The power of BLS\#2 is twice than that of BLS\#1, the coded spectrum of these two are $\left(0, \lambda_{2}, 0, \lambda_{4}, \lambda_{5}, 0,0\right)$ and $\left(2 \lambda_{1}, 0,2 \lambda_{3}, 0,2 \lambda_{5}, 0,0\right)$. The corresponding power spectral densities (PSDs) of the two users are shown in Figure 3.

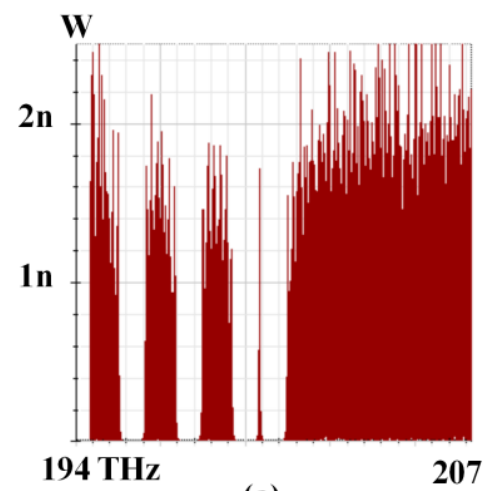

(a)

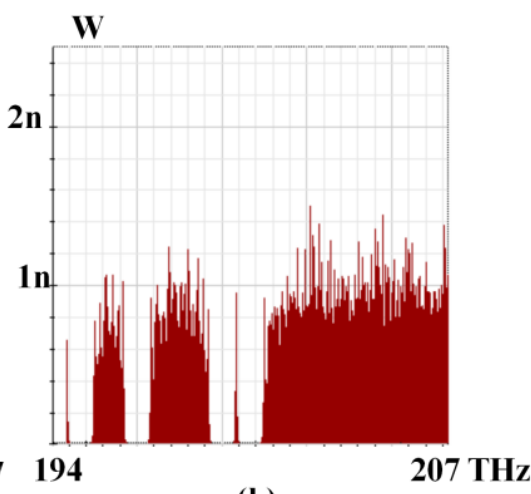

(b)

Figure 3. Downlink transmitted spectrum for user in (a) Class 2 and (b) Class 1.

In BS, a De-Mux divides the downlink signal into two parts. One part of the coded carriers is separated again by an optical splitter (OS) and sent to the decoders of both classes. Then, the passband signal from the $\mathrm{CO}$ is recovered after balanced detection and optical-to-electrical $(\mathrm{O} / \mathrm{E})$ conversion. The other part with the un-modulated carriers is split by another OS with the power ratio of 2:1. The carrier with larger power is used for the uplink transmission for Class 2. Since the optical uplink data generated from the two classes have different power levels, multi-service transmission is still supported. Here, we use one of the carriers from $\mathrm{CO}$ to achieve a wavelength scheme. Since the uplink signal is directly modulated on this carrier, it not necessary to equip an extra light source at the BS. Therefore, the burdens of cost and the system requirement at the up-link receiver are released. After being encoded by two distinct MSSP codes and combined by an OC, the uplink optical signals 
from both classes are transmitted to the CO. The passband signal is down-converted from optical to electrical domains by the decoding and photo-detecting procedures similar to the BS.

To retrieve the original data from other users, interference cancellation is performed at the decoder. In the SAC scheme, codes with fixed cross-correlation and proper decoder design remove MAI effect significantly. Correlation property between any two code vectors $\mathbf{C}_{i}$ and $\mathbf{C}_{j}$ is described as follows:

$$
\begin{aligned}
& \mathbf{C}_{i} \odot \mathbf{C}_{j}=\left\{\begin{array}{r}
p+1, i=j \\
1, i \neq j
\end{array},\right. \\
& \text { and } \mathbf{C}_{i} \odot \overline{\mathbf{C}}_{j}=\left\{\begin{array}{l}
0, i=j \\
p, i \neq j
\end{array},\right.
\end{aligned}
$$

where $\overline{\mathbf{C}}_{j}$ is the complementary code vectors of $\mathbf{C}_{j}$ and $\odot$ is the dot-product symbol. From Equations (1) and (2), we find that the MSSP code has unit correlation and can be used for designing the MAI elimination process:

$$
\mathbf{C}_{i} \odot \mathbf{C}_{j}-\frac{1}{p}\left(\mathbf{C}_{i} \odot \overline{\mathbf{C}}_{j}\right)=\left\{\begin{array}{r}
p+1, i=j \\
0, i \neq j
\end{array} .\right.
$$

\section{System Performance Analysis}

In this section, we investigate the QoS of the proposed architecture by deriving SNR expression. Since MAI is negligible in the SAC scheme, system performance is almost decided by the noise terms induced in the photo-detecting process. Thermal noise and PIIN are considered as the main noise sources. For simplicity, a two-class network with the downlink transmission only is taken in the following analysis. The photocurrent obtained at the output of balanced detector is expressed as follows [13]:

$$
I(t)=i_{k}(t)+i_{P}(t)+\overline{i_{P}}(t)+i_{T H}(t),
$$

where $i_{k}(t)$ is the current of BS \#k, $i_{P}(t)$ and $\overline{i_{P}}(t)$ are PIIN terms of the correlated and the complimentary correlated signal, and $i_{T H}(t)$ is the thermal noise. We firstly denote the transmitted spectra from all users as a time-varying vector:

$$
\mathbf{S}(t)=\frac{P_{s r}}{v}\left[\sum_{i=1}^{K_{1}} \mathrm{C}_{i}\left(\frac{1+r_{i}(t)}{2}\right)+2 \sum_{i=K_{1}+1}^{K_{1}+K_{2}} \mathrm{C}_{i}\left(\frac{1+r_{i}(t)}{2}\right)\right]
$$

where $r_{i}(t)$ is the normalized radio signal with mean $E\left[r_{i}(t)\right]=0$ and time-average power $E\left[r_{i}{ }^{2}(t)\right]=1 / 2 . P_{s r}$ and $v$ are the power and the band-width of BLS, respectively. The signal of the desired BS \#k for two classes is derived by correlation subtraction in Equation (2):

$$
\begin{aligned}
i_{k}(t) & =\frac{R P_{s r}}{M}\left[\mathbf{C}_{k} \odot \mathbf{S}(t)-\frac{1}{p} \overline{\mathbf{C}}_{\boldsymbol{k}} \odot \mathbf{S}(t)\right]=\frac{R P_{s r}}{M}\left[\left(\mathbf{C}_{k}-\frac{1}{p} \overline{\mathbf{C}}_{k}\right) \odot\left(\sum_{i=1}^{K_{1}} \mathbf{C}_{i}+2 \sum_{i=K_{1}+1}^{K_{2}} \mathbf{C}_{i}\right)\right] R_{k}(t) \\
& =\left\{\begin{array}{l}
\frac{\omega R P_{s r}}{M} R_{k}(t), \\
\frac{2 \omega R P_{s r}}{M} R_{k}(t), \quad k \in\left\{K_{1}+1, K_{2}+2, \ldots K_{2}\right\}
\end{array}\right.
\end{aligned}
$$


where $R$ is the responsivity of the photo-diode (PD), and $R_{k}(t)=\left(1+r_{k}(t)\right) / 2$. One can see that the signal amplitude of Class 2 is twice than that of Class 1 . PIIN-induced variances of the correlated signal of the complementary one in BS \#k are expressed as:

$$
\begin{aligned}
& \operatorname{Var}\left[i_{P}(t)\right]=\frac{B R^{2} P_{s r}^{2}}{M v}\left\{\mathbf{C}_{\boldsymbol{k}} \odot[\mathbf{S}(t) \otimes \mathbf{S}(t)]\right\} \\
& =\frac{B R^{2} P_{s r}^{2}}{M v}\left\{\mathbf{C}_{\boldsymbol{k}} \odot\left[\left(\sum_{i=1}^{K_{1}} \mathbf{C}_{i}+2 \sum_{i=K_{1}+1}^{K_{2}} \mathbf{C}_{i}\right) \otimes\left(\sum_{j=1}^{K_{1}} \mathbf{C}_{j}+2 \sum_{j=K_{1}+1}^{K_{2}} \mathbf{C}_{j}\right)\right]\right\} R^{2}(t) \quad \text {, and } \\
& =\left\{\begin{array}{lr}
\frac{\omega B R^{2} P_{s r}^{2}}{M^{2} v}\left(K_{1}+2 K_{2}+p\right)\left(K_{1}+2 K_{2}\right) R_{k}^{2}(t), & k \in\left\{1,2, \ldots K_{1}\right\} \\
\frac{\omega B R^{2} P_{s r}^{2}}{M^{2} v}\left(K_{1}+2 K_{2}+2 p+1\right)\left(K_{1}+2 K_{2}\right) R_{k}^{2}(t), & k \in\left\{K_{1}+1, K_{2}+2, \ldots K_{2}\right\}
\end{array}\right. \\
& \operatorname{Var}\left[i_{\bar{P}}(t)\right]=\frac{B R^{2} P_{s r}^{2}}{p^{2} M v}\left\{\overline{\mathbf{C}}_{\boldsymbol{k}} \odot[\mathbf{S}(t) \otimes \mathbf{S}(t)]\right\} \\
& =\frac{B R^{2} P_{s r}^{2}}{p^{2} M v}\left\{\overline{\mathbf{C}}_{\boldsymbol{k}} \odot\left[\left(\sum_{i=1}^{K_{1}} \mathbf{C}_{i}+2 \sum_{i=K_{1}+1}^{K_{2}} \mathbf{C}_{i}\right) \otimes\left(\sum_{j=1}^{K_{1}} \mathbf{C}_{j}+2 \sum_{j=K_{1}+1}^{K_{2}} \mathbf{C}_{j}\right)\right]\right\} R^{2}(t) \text {, } \\
& =\left\{\begin{array}{lr}
\frac{\omega B R^{2} P_{s r}^{2}}{p M^{2} v}\left(K_{1}+2 K_{2}-1\right)\left(K_{1}+2 K_{2}\right) R_{k}^{2}(t), & k \in\left\{1,2, \ldots K_{1}\right\} \\
\frac{\omega B R^{2} P_{s r}^{2}}{p M^{2} v}\left(K_{1}+2 K_{2}-2\right)\left(K_{1}+2 K_{2}\right) R_{k}^{2}(t), & k \in\left\{K_{1}+1, K_{2}+2, \ldots K_{2}\right\}
\end{array}\right.
\end{aligned}
$$

where $B$ is the electrical bandwidth, and $\otimes$ is the Kronecker product [14]. The variance of thermal noise is

$$
\operatorname{Var}\left[i_{T H}(t)\right]=S_{T H} B,
$$

where $S_{T H}$ is the power spectral density (PDF) of thermal noise. Therefore, the average SNR of the SAC-based RoF system is formulated as follows:

$$
\mathrm{SNR}=\frac{\left\{E\left[i_{k}(t)\right]\right\}^{2}}{E\left\{\operatorname{Var}\left[i_{T H}(t)\right]\right\}+E\left\{\operatorname{Var}\left[i_{P}(t)\right]\right\}+E\left\{\operatorname{Var}\left[i_{\bar{P}}(t)\right]\right\}} .
$$

Assuming that $r_{k}(t)$ is binary phase shift keying (BPSK) signal, the BER expression is:

$$
\mathrm{BER}=\frac{1}{2} \operatorname{erfc}\left(\sqrt{\frac{\mathrm{SNR}}{4}}\right)
$$

\section{Simulation Results and Discussion}

In this section, numerical analysis according to Equation (10) and simulations done by Optisystem $^{T M}$ are both demonstrated. Received user power $P$ is defined as $P_{s r}=P_{1}=P_{2} / 2$, and user numbers are $K_{1}=K_{2}=3$. Other parameters are set as follows: $B=0.6 \mathrm{GHz}, R=0.9 \mathrm{~A} / \mathrm{W}$, and $v=7 \mathrm{THz}$. A $20 \mathrm{~km}$ single mode fiber (SMF) was used to connect the CO and the BS. A 3-dB-gain erbium-doped fiber amplifier (EDFA) was employed in front of the down- and uplink receiver to compensate the insertion loss from the components of OS's and optical combiners.

BER curves for users of Class 1 and Class 2 are shown in Figure 4 . The legend of $S_{\mathrm{n}}$ denotes the user in class $n, n=1,2$. As the user power increases, lower BER values are reached. The BERs of users in Class 2 are better than those in Class 1, since the photocurrent after correlation subtraction leads to a higher SNR. For Class 1, the power penalty is $3.5 \mathrm{~dB}$ at BER $=10^{-9}$. Additionally, the simulation results nearly match the numerical ones. The small difference between these two curves are the results of the vibrations of the light source. The effects mentioned above become severe when BLS power gets large, where the light source vibrates in a larger amplitude and more power leakage occurs. Therefore, the discrepancy between software and numerical simulation is more obvious for Class 2 .

Figure 5 shows the differential BER curves according to the power margin of downlink and uplink transmissions. The performance gap between these two comes from the power losses of the system components. From the above results, one can see that the proposed power control scheme achieves differential service with different qualities of BER. 


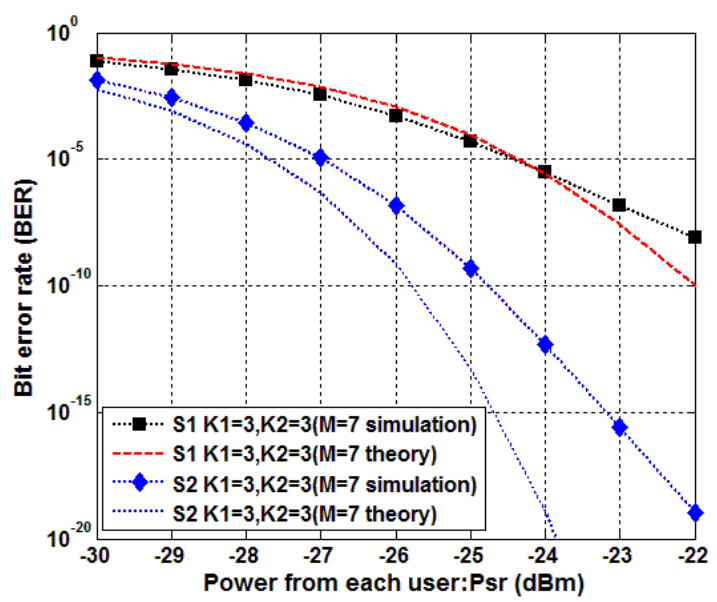

Figure 4. Bit-error rate (BER) comparison between numerical and simulation results for Classes 1 and 2.

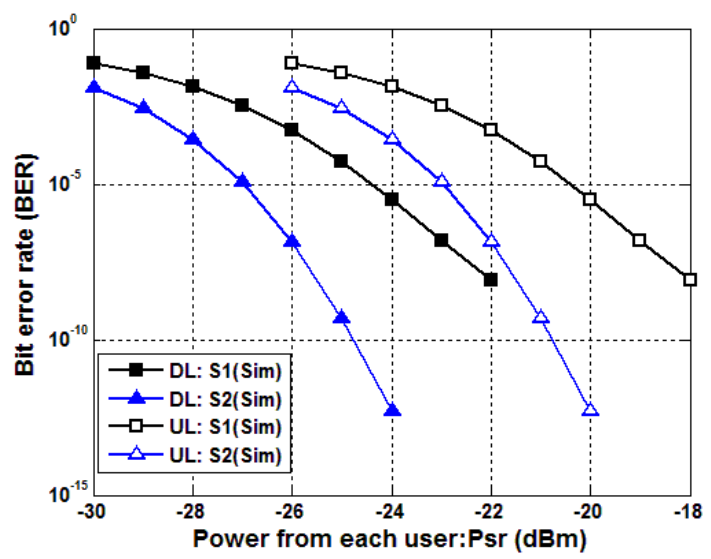

Figure 5. Bit-error rate (BER) comparison between numerical and simulation results for Classes 1 and 2.

\section{Conclusions}

In this paper, we proposed a differential RoF structure with power control based on the SAC-OCDMA system. By adopting the wavelength-reuse technique, the differential service can be accomplished without an increase in BS complexity. Users with large signal power obtain relatively small BERs without MAI. The proposed scheme provides optical networks with different performance requirements while retaining system simplicity.

Acknowledgments: This work was supported by Ministry of Science and Technology, Taiwan, project No. 104-2221-E-168-010.

Author Contributions: C.C. Yang proposed the concept and initialized the research, K.S. Chen carried out the system design and wrote the paper, J.F. Huang guided the studies; J.C. Kuo ran the simulation and analyzed the data.

Conflicts of Interest: The authors declare no conflict of interest.

\section{References}

1. Xu, Z.Z.; Wang, H.X.; Ji, Y.F. Multichannel resource allocation mechanism for $60 \mathrm{GHz}$ radio-over-fiber local access networks. J. Lightw. Technol. 2013, 5, 254-260. [CrossRef]

2. Chang, C.Y.; Yang, G.C.; Chang, C.Y.; Kwong, W.C. Study of a diversity O-CDMA scheme for optical wireless. J. Lightw. Technol. 2012, 30, 1549-1558. [CrossRef]

3. Yang, C.C.; Huang, J.F.; Chang, H.H.; Chen, K.S. Radio transmissions over residue-stuffed-QC-coded optical CDMA network. IEEE Commun. Lett. 2014, 18, 329-331. [CrossRef] 
4. Yang, C.C. Optical CDMA-based fiber-radio networks with improved power efficiency. IEEE Trans. Commun. 2012, 60, 810-816. [CrossRef]

5. Ahmed, M.; Ahmad, I.; Habibi, D. Service class resource management for green wireless-optical broadband access networks (WOBAN). J. Lightw. Technol. 2015, 33, 7-18. [CrossRef]

6. Zhu, Q.Y.; Pavel, L. Enabling differentiated services using generalized power control model in optical networks. IEEE Trans. Commun. 2009, 57, 2570-2575.

7. Matsuura, M.; Oki, E. Optical carrier regeneration for carrier wavelength reuse in a multicarrier distributed WDM network. IEEE Photon. Technol. Lett. 2010, 22, 808-810. [CrossRef]

8. Cui, W.T.; Shao, T.; Yao, J.P. Wavelength reuse in a UWB over fiber system based on phase-modulation to intensity-modulation conversion and destructive interferencing. J. Lightw. Technol. 2013, 31, 2904-2912. [CrossRef]

9. Kaszubowska, L.H.; Barry, L. Remote down conversion with wavelength reuse for the radio/fiber uplink connection. IEEE Photon. Technol. Lett. 2006, 18, 562-564. [CrossRef]

10. Attygalle, M.; Lim, C.; Pendock, P.J.; Nirmalathas, A.; Edvell, G. Transmission improvement in fiber wireless links using fiber bragg gratings. IEEE Photon. Technol. Lett. 2005, 17, 190-192. [CrossRef]

11. Chen, K.S.; Yang, C.C.; Huang, J.F. Using stuffed quadratic congruence codes for SAC labels in optical packet switching network. IEEE Commun. Lett. 2015, 19, 1093-1096. [CrossRef]

12. Yang, C.C. Optical CDMA passive optical network using prime code with interference elimination. IEEE Photon. Technol. Lett. 2007, 19, 516-518. [CrossRef]

13. Noshad, M.; Jamshidi, K. Bounds for the BER of codes with fixed cross correlation in SAC-OCDMA Systems. J. Lightw. Technol. 2011, 29, 1944-1950. [CrossRef]

14. Horn, R.A.; Johnson, C.R. Matrix Analysis, 2nd ed.; Cambridge University Press: Cambridge, UK, 2012.

(C) 2016 by the authors; licensee MDPI, Basel, Switzerland. This article is an open access article distributed under the terms and conditions of the Creative Commons Attribution (CC-BY) license (http://creativecommons.org/licenses/by/4.0/). 